\title{
FRIEDMAN UNIVERSE WITH THE COSMOLOGICAL CONSTANT
}

\author{
M. P. Korkina, E. M. Kopteva \\ Department of Physics, Dnipropetrovsk National University \\ 13 Naukoviy Per., Dnipropetrovsk, 49050, Ukraine
}

(Received July 31, 2002)

\begin{abstract}
Inhomogeneous dust distribution in the presence of the cosmological constant $(\Lambda)$ is considered. The exact solution to the Einstein equations generalizing the Tolman solution for the parabolic motion of the dust, and the solution describing FRW-like Universe with nonzero cosmological constant, are studied.
\end{abstract}

Key words: universe, Einstein equations, cosmological constant.

PACS number(s): 04.20.Jb, 04.20.Gv, 98.80.Es

\section{INTRODUCTION}

According to the last sufficiently precise observational data the cosmological constant plays an important dynamical role in our Universe. Among the experiments being evidence of above there are the observations on supernova, studying the temperature anisotropies in the cosmic microwave background and using the method of estimation the mass density by "weighing" clusters of galaxies [1-6]. The most severe constrains on the matter density and the value of cosmological constant are given by the observational data concerning the supernova and cosmic microwave background. Especially one should mention the experimental results of the year 2000 . These data exclude the existence of closed Universe while preferring flat open models. As far as the problem of the vacuum energy is one of the most fundamental problems of theoretical physics, it is also very important to note that the cosmological constant can be treated as the vacuum energy. On this basis it seems to be interesting to consider the astrophysical and cosmological models in the presence of the cosmological constant. Taking into account the fact that the radiation prevailed only in early Universe, we chose for now the metric description of the dust matter distribution.

\section{TOLMAN SOLUTION WITH THE COSMOLOGICAL CONSTANT}

Let us take the metric in synchronous coordinates. For the dust matter this coordinate system is a comoving system. And for the vacuum with the state equation $\varepsilon+p=0$ any coordinate system plays the role of a comoving system.

The metric interval is represented by

$$
d s^{2}=c^{2} d t^{2}-\frac{r^{\prime 2} d R^{2}}{f^{2}(R)}-r^{2}(R, t)\left(d \theta^{2}+\sin ^{2} \theta d \varphi^{2}\right)
$$

where $r^{\prime}=\partial r / \partial R$ and $f(R)$ is an arbitrary function of integration. The function $f(R)$ determines the total energy of the dust particles in a shell R: $f(R)=E(R) / m c^{2}$.
Let us consider the parabolic motion $f(R)=1$. For homogeneous and isotropic models this case corresponds to a flat space. From the Einstein equations and metrie (1) for the case under consideration we have

$$
\dot{r}^{2}(R, t)=\frac{m(R)}{r(R, t)}+\Lambda r^{2}(R, t)
$$

here $\dot{r}(R, t)=\partial r / \partial t$ and $m(R)$ is a mass function which determines the total mass of a dust ball with the radius $\mathrm{R}$ (in length units)

$$
m^{\prime}(R)=\frac{8 \pi \gamma}{c^{4}} \varepsilon(R, t) r^{2} r^{\prime}
$$

Making the replacement

$$
r(R, t)=\psi^{2 / 3}(R, t)
$$

we will obtain

$$
\frac{9}{4} \dot{\psi}^{2}(R, t)=m(R)+\psi^{2}(R, t)
$$

This equation has the exact analytical solution from which we have

$$
r(R, t)=\left(\frac{m(R)}{\Lambda}\right)^{1 / 3}\left[\sinh \frac{3}{2} c \sqrt{\Lambda}\left(t_{0}(R)-t\right)\right]^{2 / 3}
$$

for the case of collapse $\left(-\infty<t<t_{0}\right)$, and

$$
r(R, t)=\left(\frac{m(R)}{\Lambda}\right)^{1 / 3}\left[\sinh \frac{3}{2} c \sqrt{\Lambda}\left(t-t_{0}(R)\right)\right]^{2 / 3}
$$

for the case of expansion $\left(t_{0}<t<\infty\right)$.

$t_{0}(R)$ is another arbitrary function of integration which defines either the time of collapse (for solution (6)) or the start time of expansion (for solution (7)), it is special for each shell $R$. The obtained solutions (6) and (7) represent the generalization of well-known Tolman 
solution [7] to the case of nonzero cosmological constant. Indeed within the limit of $\Lambda \rightarrow 0$ these solutions turn into correspondent Tolman solutions for the collapsing and expanding spherically symmetric dust:

$$
r_{T}(R, t)=\left[ \pm \frac{3}{2} c \sqrt{m(R)}\left(t_{0}(R)-t\right)\right]^{2 / 3} .
$$

Studying the behaviour of (6) and (7) in the limit of small $\left(t_{0}(R)-t\right)$ we will obtain the same results as if it came from (8). Thus the cosmological constant does not play any role at the beginning of the expansion nor near the collapse singularity.

Under the condition

$$
t \gg t_{0}(R)+\frac{1}{3 c \sqrt{\Lambda}}
$$

solution (7) can be written as

$$
r_{s}(R, t)=\left(\frac{m(R)}{4 \Lambda}\right)^{1 / 3} \exp \left[c \sqrt{\Lambda}\left(t-t_{0}(R)\right)\right]
$$

It represents the de Sitter metric [7] and turns into Lemaitre-Robertson solution under $m(R) \sim R^{3}$ and $t_{0}(R)=0[7]$.

\section{FRIEDMAN SOLUTION WITH THE COSMOLOGICAL CONSTANT}

For homogeneously and isotropically distributed dust matter under condition $f^{2}(R)=1$ we have $r=R a(t)$, $t_{0}(R)=0$ and the interval will take the form:

$$
d s^{2}=c^{2} d t^{2}-a^{2}(t)\left(d R^{2}+R^{2} d \sigma^{2}\right)
$$

hence this is a flat model. Since energy density has only dependence on time it follows from (3) that

$$
\frac{m(R)}{R^{3}}=\frac{8 \pi \gamma}{3 c^{4}} \varepsilon(t) a^{3}(t)=\frac{1}{a_{0}^{2}},
$$

here $a_{0}$ is a dimensionful constant with units of length. In this case

$$
m(R)=R^{3} a_{0}^{-2}
$$

Using (7) and (13) we can write the scale factor for the homogeneous and isotropic flat Universe:

$$
a(t)=\left[\frac{a_{\Lambda}}{a_{0}} \sinh \frac{3}{2} \frac{c t}{a_{\Lambda}}\right]^{2 / 3} .
$$

This expression was discussed in paper [10], and correspondent solution at first was obtained by Lemaitre (1927). Here we have introduced the constant $a_{\Lambda}=$ $\Lambda^{-1 / 2}$ which is a minimal radius of normal section of 4-dimensional hyperboloid embedded into a flat 5dimensional space. This hyperboloid represents the de Sitter space. Similarly to the generalized Tolmen solution the solution (14) under small $t(t \rightarrow 0)$ goes to the ordinary Friedman solution, and in the limit of great $t$ turns into the Lemaitre-Robertson one which describes the de Sitter space and corresponds to parabolic sections of 4-dimensional hyperboloid [8].

Let us find the density parameters of the dust matter and vacuum [1]. From (12) we have for the matter energy density:

$$
\varepsilon_{M}(t)=\frac{3 c^{4}}{8 \pi \gamma a_{0^{2}}} \frac{1}{a^{3}(t)}
$$

the vacuum energy density is given by

$$
\varepsilon_{\Lambda}=\frac{3 c^{4} \Lambda}{8 \pi \gamma}
$$

and for the critical energy density we have

$$
\varepsilon_{\mathrm{cr}}=\frac{3 c^{4}}{8 p \gamma} \frac{\dot{a}^{2}}{a^{2}}
$$

From here we can write the density parameters:

$$
\begin{gathered}
\Omega_{M}=\frac{\varepsilon_{M}}{\varepsilon_{c r}}=\cosh ^{-2} \frac{3}{2} \frac{c t}{a_{\Lambda}}, \\
\Omega_{\Lambda}=\frac{\varepsilon_{\Lambda}}{\varepsilon_{c r}}=\tanh ^{2} \frac{3}{2} \frac{c t}{a_{\Lambda}},
\end{gathered}
$$

and deceleration parameter for the Universe expansion is given by

$$
q=\frac{2-\cosh 3 c t / a_{\Lambda}}{1+\cosh 3 c t / a_{\Lambda}}
$$

Under condition

$$
t=t_{\Lambda}=\frac{a_{\Lambda}}{3 c} \ln (2+\sqrt{3})
$$

parameter $q$ goes to zero. Taking into account the fact that $\Omega_{\Lambda}+\Omega_{M}=1$ (since we consider the flat Universe) we can find the point of zero Universe expansion acceleration, it turns to be $\Omega_{\Lambda}=1 / 3, \Omega_{M}=2 / 3$. When $t<t_{\Lambda}$ the expansion is decelerated, i.e., Universe with the scale factor (14) behaves similarly to the FriedmanRobertson-Walker (FRW) model (with no $\Lambda$ ). Under $t=t_{\Lambda}$ deceleration becomes an acceleration, which grows 
exponentially with time. One should note that all the parameters depend only on cosmological term (contain $a_{\Lambda}$ ) and not on the matter characteristics (do not include $a_{0}$ ).

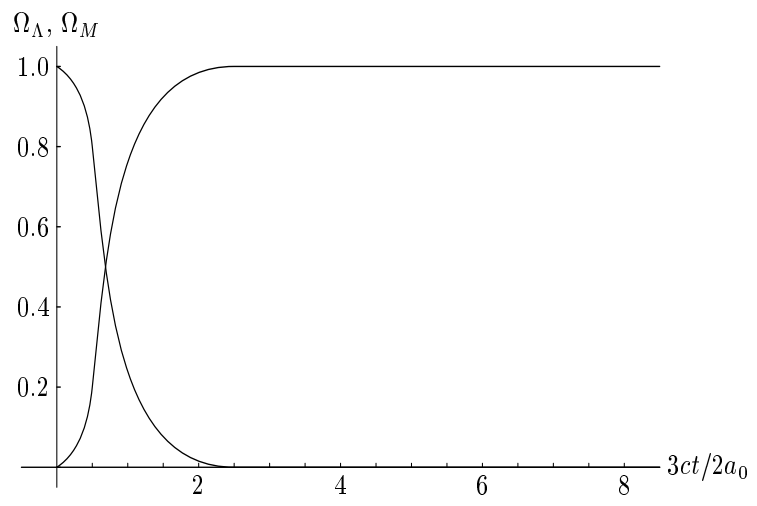

Fig. 1.

Fig. 1 shows $\Omega_{\Lambda}$ and $\Omega_{M}$ dependence on time. At the start moment $(t=0) \Omega_{M}=1\left(\Omega_{M}\right.$ always equals unity when $\Lambda$ is absent). Then $\Omega_{M}$ decreases with time, and at the limit of $t \rightarrow \infty \Omega_{M} \rightarrow 0$. $\Omega_{\Lambda}$ grows with time from zero (when $t=0$ ) to unity (when $t \rightarrow \infty$ ). According to recent data $\Omega_{M} \approx 0.3$ and $\Omega_{\Lambda} \approx 0.7[1,2]$. This corresponds to the fact that

$$
\frac{t_{n}}{a_{\Lambda}} \approx 2.7 \cdot 10^{-11} \mathrm{~cm}^{-1} \mathrm{sec}
$$

here $t_{n}$ represents up-to-date time, and if we take $a_{\Lambda} \approx$ $10^{28} \mathrm{~cm}$. Then

$$
t_{n} \approx 2.7 \cdot 10^{17} \mathrm{sec}
$$

Comparing $t_{n}$ with the time of changing deceleration into acceleration $t_{\Lambda}(21)\left(t_{\Lambda} \approx 1.5 \cdot 10^{17} \mathrm{sec}\right)$ one can see that $t_{n}=1.08 \cdot t_{\Lambda}$, i.e., at present deceleration has already changed into acceleration.

\section{CONCLUSIONS}

An exact solution for the inhomogeneous distribution of the dust matter with nonzero cosmological constant is obtained and investigated. The parabolic motion of the dust particles is considered. It is shown that the obtained solution represents generalization of the Tolman solution for the case of nonzero cosmological constant. It is shown that near the singularity solution (6) turns into the Tolman solution and goes to the de Sitter one at late times. The solution for the homogeneous dust matter with flat space is found. This solution describes the FRW-like Universe in the presence of the cosmological constant (14). The energy density parameters for the matter and vacuum are calculated, and the deceleration parameter for the expansion of our Universe is found. We also demonstrate that all these parameters depend only on the cosmological constant and nor on the dust matter characteristics. The density parameters dependence on time is plotted
[1] S. Carroll, preprint astro-ph/00004075.

[2] P. D. Mannheim, preprint gr-qc/ 9903005.

[3] P. de Bernardis, P. A. R. Ade, J. J. Bock et al., Nature 404, 955 (2000)

[4] S. Perlmutter et al., Astrophys. J. 517, 565 (1990); preprint astro-ph/9812133.

[5] J. R. Bond, A. H. Jaffe, L. Knox, Phys. Rev. D 57, 2117 (1988); preprint astro-ph/9708203.

[6] J. R. Gott, M. G. Park, H. M. Lee, Astrophys.. J. 338,
1 (1989).

[7] R. C. Tolman, Relativvity Thermodinamics and Cosmology (Oxford, Clarendon Press, 1969).

[8] S. W. Hawking, G. F. R. Ellis, The Large Scale Stucture of Spase-Time (Cambridge University Press, 1973).

[9] J. R. Oppenheimer, H. Snyder, Phys. Rev. 56, 455 (1939).

[10] V. R. Gavrilov, V. N. Melnikov, preprint gr-qc/0101005.

\title{
ФРІДМАНІВСЬКИЙ ВСЕСВІТ 3 КОСМОЛОГІЧНОЮ СТАЛОЮ
}

\author{
М. П. Коркіна ${ }^{1}$, Е. М. Коптєва ${ }^{2}$ \\ Дніпропетровсъкий начіональний університет, фізичний факультет, \\ Науковий пров., 13, Дніпропетровсък, 49050, Украӥна \\ email: ${ }^{1}$ korkina@ff.dsu.dp.ua; ${ }^{2}$ kopteva@ff.dsu.dp.ua
}

Розглянуто неоднорідний розподіл пилу при наявності космологічної сталої $\Lambda$. Вивчено точний розв'язок рівнянь Айнштайна, який узагальнює розв'язок Толмена для параболічного руху пилу, i розв'язок з ненульовою космологічною постійною, який описує Всесвіт Фрідмана-Робертсона-Уокера. 